\title{
Pilot evaluation of an enzymatic assay for rapid measurement of antiretroviral drug concentrations
}

\author{
Ayokunle O. Olanrewaju', Benjamin P. Sullivan', Ashley R. Bardon², Tiffany J. Lo ${ }^{3}$, Tim R. Cressey ${ }^{4,5,6}$, \\ Jonathan D. Posner ${ }^{1,7,8}$ and Paul K. Drain ${ }^{2,9,10^{*}}$ (B)
}

\begin{abstract}
Objective: Maintaining adequate drug adherence is crucial to ensure the HIV prevention benefits of pre-exposure prophylaxis (PrEP). We developed an enzymatic assay for rapidly measuring tenofovir-diphosphate (TFV-DP) concentrations - a metabolite that indicates long-term PrEP adherence.

Setting: The study was conducted at the Madison HIV Clinic at Harborview Medical Center in Seattle.

Methods: We enrolled adults receiving standard oral PrEP, and individuals not receiving any antiretrovirals. We measured TFV-DP concentrations in diluted whole blood using our novel REverSe TRanscrlptase Chain Termination (RESTRICT) assay, based on inhibition of HIV reverse transcriptase (RT) enzyme. Blood samples were diluted in water, DNA templates, nucleotides, RT, and intercalating dye added, and results measured with a fluorescence readerstronger fluorescence indicated higher RT activity. We compared RESTRICT assay results to TFV-DP concentrations from matched dried blood spot samples measured by liquid chromatography tandem mass spectrometry (LC-MS/ MS) using $\geq 700 \mathrm{fmol} /$ punch TFV-DP as a threshold for adequate adherence ( $\geq 4$ doses/week).

Results: Among 18 adults enrolled, 4 of 7 participants receiving PrEP had TFV-DP levels $\geq 700 \mathrm{fmol} /$ punch by LC-MS/ MS. RESTRICT fluorescence correlated with LC-MS/MS measurements $(r=-0.845, p<0.0001)$. Median fluorescence was 93.3 (95\% confidence interval [Cl] 90.9 to 114) for samples $<700 \mathrm{fmol} /$ punch and 54.4 (Cl 38.0 to 72.0 ) for samples $\geq 700 \mathrm{fmol} /$ punch. When calibrated to an a priori defined threshold of 82.7, RESTRICT distinguished both groups with $100 \%$ sensitivity and $92.9 \%$ specificity.
\end{abstract}

Conclusions: This novel enzymatic assay for measuring HIV reverse transcriptase activity may be suitable for distinguishing TFV-DP concentrations in blood that correspond to protective PrEP adherence.

Keywords: Adherence, Pre-exposure prophylaxis, Enzymatic assay, Tenofovir diphosphate

\section{Introduction}

Pre-exposure prophylaxis (PrEP) can prevent HIV acquisition [1,2], and maintaining adequate adherence is critical for PrEP efficacy [3-5]. In several PrEP trials

*Correspondence: pkdrain@uw.edu

${ }^{2}$ Department of Global Health, Schools of Medicine and Public Health, University of Washington, Seattle, USA

Full list of author information is available at the end of the article and implementation studies, PrEP clients had difficulties maintaining adequate adherence and persistence, and monitoring their PrEP use was challenging $[4,6,7]$. Various indirect and subjective measures have been used to measure adherence [8-11], while quantifying concentrations of HIV drugs in clinical samples may provide more objective information for adherence measurement $[8$, 12-14]. original author(s) and the source, provide a link to the Creative Commons licence, and indicate if changes were made. The images or other third party material in this article are included in the article's Creative Commons licence, unless indicated otherwise in a credit line to the material. If material is not included in the article's Creative Commons licence and your intended use is not permitted by statutory regulation or exceeds the permitted use, you will need to obtain permission directly from the copyright holder. To view a copy of this licence, visit http://creativecommons.org/licenses/by/4.0/. The Creative Commons Public Domain Dedication waiver (http://creativeco mmons.org/publicdomain/zero/1.0/) applies to the data made available in this article, unless otherwise stated in a credit line to the data. 
Tenofovir disoproxil fumarate (TDF), which is used in oral PrEP regimens [15], is hydrolyzed in the blood into tenofovir (TFV) and phosphorylated intracellularly into tenofovir diphosphate (TFV-DP) [16]. TFV has a short half-life $(15 \mathrm{~h})$ and can be detected for up to 7 days in plasma after TDF ingestion [17]. TFV has higher and more variable concentrations in urine and can be detected up to 14 days after TDF ingestion [18, 19]. TFV measurement is susceptible to the "white coat" effect where one is unable to distinguish recent pill ingestion from patterns of long term adherence. Intracellular TFV-DP has a longer half-life as it accumulates in red blood cells (RBCs) and peripheral blood mononuclear cells, and can provide a window into cumulative adherence over 1-2 months [20]. Directly observed therapy trials were used to establish that a TFV-DP concentration in dried blood spot (DBS) samples $\geq 700 \mathrm{fmol} / 3 \mathrm{~mm}$ punch is equivalent to $\geq 4$ TDF doses/week on average and provides adequate reduction of HIV incidence risk in the context of PrEP, while TFV-DP $\geq 1250 \mathrm{fmol} /$ punch indicates perfect (7 doses/week) adherence among men who have sex with men receiving PrEP [12, 21]. Measurement of TFV-DP in DBS samples is performed using liquid chromatography tandem mass spectrometry (LCMS/MS) and although these assays provide accurate and quantitative results, they are expensive, laborious, and may be unsuitable for routine clinical use.

We recently developed an enzymatic assay, termed REverSe TRanscrIptase Chain Termination (RESTRICT), for rapid measurement of HIV reverse transcriptase activity, as a proxy measure of intracellular TFV-DP concentrations [22]. RESTRICT infers TFV-DP levels in a blood samples based on the extent of DNA synthesis by recombinant HIV RT using DNA templates, primers, nucleotides, and intercalating dyes provided during the assay. Our initial results with RESTRICT showed that we can accurately distinguish TFV-DP concentrations spiked into blood corresponding to low and high PrEP adherence [22]. In this study, we compared the RESTRICT assay with TFV-DP measurement by LC-MS/MS among a cohort of adults receiving oral PrEP in Seattle.

\section{Methods}

\section{Study participants}

We enrolled adults receiving oral PrEP (TDF + emtricitabine) and individuals not receiving any HIV medications at the Madison Clinic at Harborview Medical Center in Seattle. Exclusion criteria were age under 18 years, seropositivity for HIV or flavivirus (Zika, Dengue, West Nile, Yellow Fever), or previous enrollment in HIV or flavivirus vaccine study. We collected participant data on HIV status, sociodemographic characteristics, and body mass index (BMI). All study participants were enrolled and sampled in accordance with the University of Washington/Fred Hutch Center for AIDS Research (CFAR) Enhanced Data and Specimen Collection Service. All participants provided informed consent and samples were collected in association with study identifiers.

\section{Blood sample collection and LC-MS/MS measurement}

Venous whole blood was collected from each study participant. Dried blood spot (DBS) cards were prepared using $25 \mu \mathrm{L}$ of each whole blood sample. Whole blood tubes were stored on ice and analyzed by RESTRICT within $4 \mathrm{~h}$ of sample collection. Matched whole blood and DBS samples were tested using the RESTRICT assay and LC-MS/MS. DBS cards were stored at -70 to $-80{ }^{\circ} \mathrm{C}$ until analysis. TFV-DP concentrations were measured using a validated LC-MS/MS assay in accordance with the Clinical Pharmacology Quality Assurance and Quality Control Program validation guidelines [23].

\section{RESTRICT assay principle and workflow}

RESTRICT detects TFV-DP drug concentrations based on its mechanism of action on HIV RT [22]. Fluorescence output from in vitro DNA synthesis by recombinant HIV RT is used to estimate TFV-DP concentration in a patient's blood. High fluorescence and high RT activity indicate low TFV-DP concentrations and vice-versa.

Reactions were carried out in a buffer containing: $60 \mathrm{mM}$ Tris (77-86-1, Sigma, St. Louis, MO), $30 \mathrm{mM} \mathrm{KCl}$ (7447-40-7, Sigma, St. Louis, MO), $8 \mathrm{mM} \mathrm{MgCl}_{2}$ (778630-3, Sigma, St. Louis, MO), $10 \mathrm{mM}$ dithiothreitol (20265, Sigma, St. Louis, MO), $400 \mathrm{nM}$ deoxynucleotide triphosphates (dNTPs) (D7295, Sigma, St. Louis, MO), $40 \mathrm{nM}$ primer $16 \mathrm{~S}$ rRNA Forward primer AGA GTT TGA TCC TGG CTC AG (51-01-19-06, Integrated DNA Technologies, Coralville, IA) and $4 \mathrm{nM}$ DNA template buffered to $\mathrm{pH} 8.0$ using $\mathrm{HCl}$ (7647-01-0, Acros Organics, Fair Lawn, NJ). Custom-designed DNA templates were synthesized in silico (Integrated DNA Technologies, Coralville IA) and consisted of a 20-nucleotide primer binding site followed by a 180-nucleotide T-rich detection region consisting of TTCA repeats to increase the likelihood of chain termination by TFV-DP (TTC ATTCAT TCATTCATTCAT TCATTCATTCAT TCA TTCATTCAT TCATTCATTCAT TCATTCATTCAT TCATTCATTCAT TCATTCATTCAT TCATTCATT CAT TCATTCATTCAT TCATTCATTCAT TCATTC ATTCAT TCATTCAT TCAT TCATTCATTCAT TCA TTCATTCATTCACTGAGCCAGGATCAAACTCT). Recombinant RT was obtained through the NIH AIDS Reagent Program, Division of AIDS, NIAID, NIH: HIV-1 RT Catalog \#3555 from Dr. Stuart Le Grice and Dr. Jennifer T. Miller [24]. 
RESTRICT was conducted in 5 steps (Fig. 1). First, blood samples were collected from study participants at the CFAR. Next, venous blood was diluted to $8 \%$ volume in nuclease-free water (3098, Sigma-Aldrich, St. Louis, MO) and vortexed for $5 \mathrm{~min}$ to lyse red blood cells (RBCs), release intracellular TFV-DP, and reduce assay inhibition by blood components. Then, $5 \mu \mathrm{L}$ of diluted whole blood was added to $30 \mu \mathrm{L}$ of buffered master mix in flat-bottom polystyrene 384-well plates with nonbinding surfaces (3575, Corning, Corning, NY). $5 \mu \mathrm{L}$ of HIV-1 RT at a final enzyme concentration of $100 \mathrm{nM}$ was added as the last reagent to initiate DNA synthesis and incubated at $37^{\circ} \mathrm{C}$ for $30 \mathrm{~min}$ in a microplate reader (SpectraMax iD3, Molecular Devices, San Jose, CA). Finally, PicoGreen $^{\text {TM }}$ dye (P7581, ThermoFisher Scientific, Waltham, MA) diluted 1:400 in 1 $\times$ TE (10128-588, VWR, Radnor, PA) was added to stop the reaction and provide fluorescence output. Five replicates were tested for each sample.

A standard curve was generated with five aliquots of TFV-DP spiked into diluted blood (from participant 007, not on PrEP) at final concentrations corresponding to 8.9 to $58,333 \mathrm{fmol} /$ punch in ninefold increments and spanning nearly two orders of magnitude above and below the PrEP adherence clinical range.

\section{Statistical analysis}

Baseline correction was carried out by subtracting the fluorescence obtained from each sample without added $\mathrm{RT}$ enzyme from the endpoint assay fluorescence after 30 min RT incubation. The fluorescence intensity from each sample was normalized by dividing by the average fluorescence obtained from blood samples without detectable TFV-DP by LC-MS. We calculated the Pearson correlation coefficient between RESTRICT fluorescence and LC-MS/MS TFV-DP concentrations. We compared the fluorescence of samples at thresholds for adequate adherence $(700 \mathrm{fmol} /$ punch i.e., 4 doses/week) and perfect adherence $(1250 \mathrm{fmol} /$ punch i.e., 7 doses/ week) among men who have sex with men receiving PrEP. We established a priori thresholds for fluorescence at $700 \mathrm{fmol} /$ punch and $1250 \mathrm{fmol} /$ punch by interpolating standard curves obtained by spiking known concentrations of TFV-DP in blood using GraphPad Prism.

\section{Results}

A total of 18 individuals were included [4 (22\%) women, median age 56 years; interquartile range (IQR) 48 to 56] (Table 1). All 11 participants not receiving PrEP had undetectable $(<200 \mathrm{fmol} /$ punch $)$ TFV-DP by LC-MS/ MS (Table 1). Six out of seven participants receiving PrEP had detectable TFV-DP, four out of seven participants had TFV-DP $\geq 700 \mathrm{fmol} /$ punch, and three out of seven participants had TFV-DP $\geq 1250 \mathrm{fmol} / \mathrm{punch}$.

Median fluorescence was $93.3 \%$ (95\% Confidence Interval [CI] 90.9 to 114) for samples containing $<700 \mathrm{fmol} /$ punch and $54.4 \%$ (CI 38.0 to 72.0 ) for samples containing $\geq 700 \mathrm{fmol} /$ punch. Median fluorescence was $92.5 \%$ (CI 90.9 to 109) for samples containing < $1250 \mathrm{fmol} /$ punch and $50.8 \%$ (CI 38.0 to 58.0) for samples containing $\geq 1250 \mathrm{fmol} /$ punch. We

\begin{tabular}{|c|c|c|}
\hline & $\operatorname{PrEP}(\mathrm{N}=7)$ & No $\operatorname{PrEP}(\mathrm{N}=11)$ \\
\hline Median age (IQR) & 50 (45 to 62$)$ & 57 (52 to 65$)$ \\
\hline Body mass index, BMI $\left(\mathrm{kg} / \mathrm{m}^{2}\right)$ & 25 (23 to 27 ) & 31 (23 to 37) \\
\hline Number of women (\%) & $1(14 \%)$ & $3(27 \%)$ \\
\hline $\begin{array}{l}\text { LC-MS TFV-DP concentration } \\
\text { (fmol/punch) }\end{array}$ & $\begin{array}{l}\text { 717, 2248, 2453, 2556, } \\
675,559, \text { undetect- } \\
\text { able }\end{array}$ & All undetectable \\
\hline
\end{tabular}

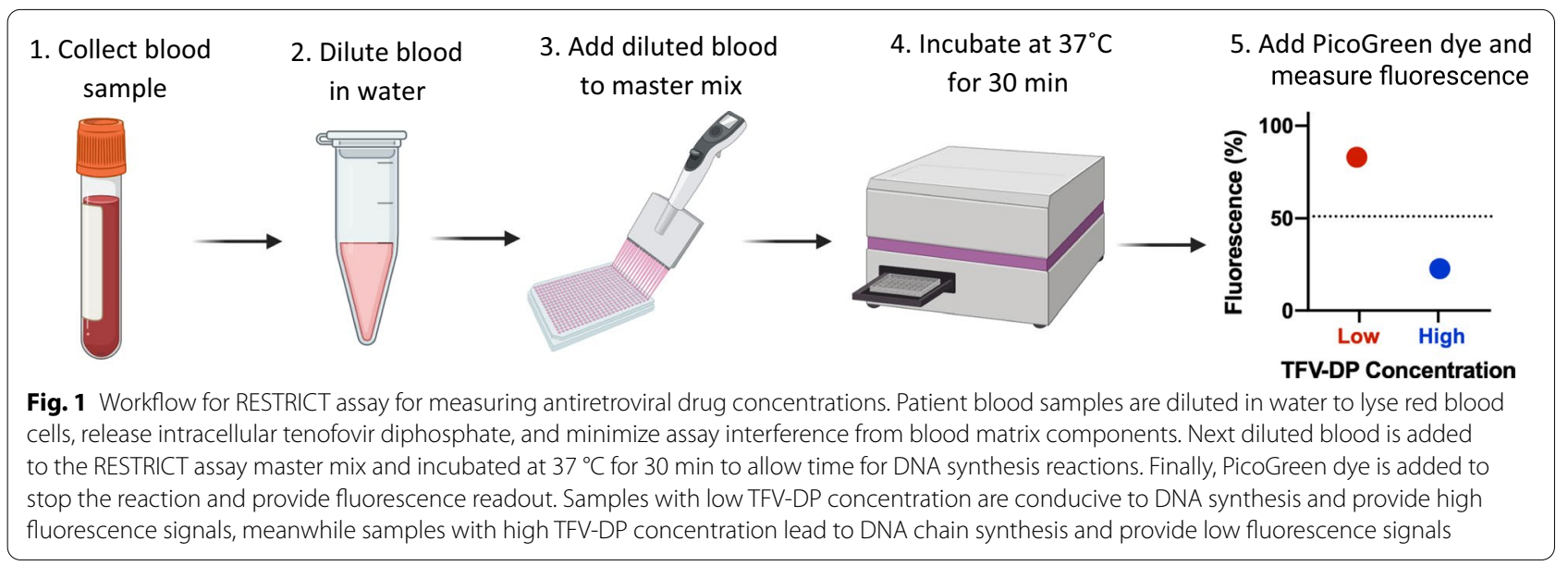


determined an a priori cut-off of $82.7 \%$ (CI 76.9 to 88.5 ) corresponding to $700 \mathrm{fmol} /$ punch by interpolating the standard curve obtained with spiked TFV-DP (Fig. 2a). Applying the a priori fluorescence threshold of $82.7 \%$ yielded $100 \%$ sensitivity and $92.9 \%$ specificity in identifying participants with TFV-DP concentrations $\geq 700 \mathrm{fmol} /$ punch, $p=0.0029$ (Fig. 2b). Similarly, we established an a priori threshold of $71.0 \%$ (CI 64.6 to 77.4) corresponding to $1250 \mathrm{fmol} /$ punch (Fig. 2a) that distinguished participants above or below the threshold with $100 \%$ specificity and $100 \%$ sensitivity (Fig. 2c). RESTRICT fluorescence intensities were correlated with LC-MS/MS measurements, $\mathrm{r}=-0.845$ (CI -0.941 to -0.624$), \mathrm{R}^{2}=0.714, p<0.0001$ (Fig. $2 \mathrm{~d}$ ).

\section{Discussion}

We developed a novel enzymatic assay (RESTRICT) to measure antiretroviral drug concentrations based on the inhibition of HIV reverse transcriptase activity. RESTRICT results correlate with TFV-DP concentrations in DBS samples quantified by LC-MS/MS. Fluorescence levels were significantly lower in individuals with TFV-DP concentrations above the threshold for adequate PrEP adherence ( $\geq 700 \mathrm{fmol} /$ punch) compared with individuals with lower or undetectable TFV-DP concentrations. In this pilot study, the RESTRICT assay was able to distinguish all 4 individuals with drug levels above the threshold for adequate adherence. RESTRICT also correctly identified 13 out of 14 samples as having drug concentrations below the threshold. The one sample that was incorrectly classified had a TFV-DP concentration of $675 \mathrm{fmol} /$ punch, which was very close to the cut-off. Similarly RESTRICT correctly identified all 3 samples from study participants with TFV-DP concentrations indicative of perfect PrEP adherence among men who have sex with men. Taken together our results suggest that RESTRICT is a useful qualitative test to distinguish between individuals with TFV-DP corresponding to adequate or perfect PrEP adherence.

Measuring antiretroviral concentrations provides accurate long-term adherence information that is correlated with clinical outcomes [12, 25, 26]. Urine TFV tests have been developed by our group and others for rapid adherence measurement $[18,26-28]$. Urine can be collected quickly and non-invasively (unlike blood samples) but urine TFV tests only measure recent medication ingestion and can be subject to white-coat pill taking [26, 29]. TFV-DP concentrations in RBCs indicate long term adherence and can be measured using LC-MS/MS; however, LC-MS/MS is complex, time-consuming, and expensive. The RESTRICT assay represents a new class of rapid and objective measure of antiretroviral drug concentrations that can be completed using reagents and

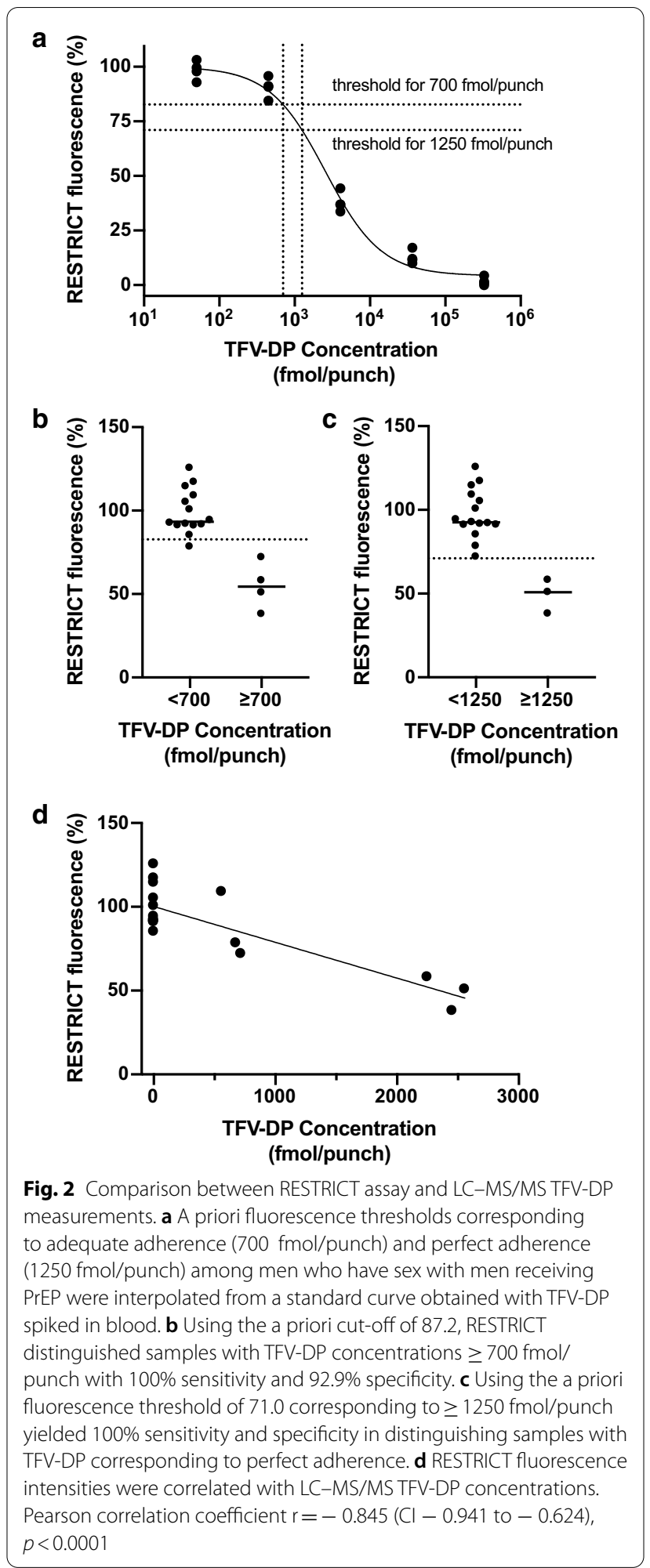

equipment that are available in most clinical laboratories and is compatible with integration into a near-patient or point-of-care format. 
Two limitations of our study are the variation in fluorescence intensities in blood samples with undetectable TFV-DP concentration (Fig. 2c) and the small sample size $(\mathrm{N}=18,7$ on PrEP). The coefficient of variation of the RESTRICT assay with blood samples with undetectable TFV-DP $(\mathrm{N}=11)$ was $13.5 \%$ compared to only $4 \%$ in buffer [22]. This variation in fluorescence intensity for samples with undetectable TFV-DP arises because blood dilution decreases but does not completely eliminate non-specific inhibition of HIV RT by blood matrix components such as hemoglobin and immunoglobulins [30]. We are investigating other user-friendly sample preparation to further decrease non-specific RT inhibition and assay variation in blood [31]. While additional data is required to more rigorously compare RESTRICT and LC-MS/MS measurements, our findings here and our previous work with spiked blood samples[22] provide preliminary evidence for the potential of the RESTRICT assay for rapid detection of antiretroviral drug concentrations in clinical settings.

Ongoing work is aimed at gathering more data to validate the RESTRICT assay with blood samples from both PrEP clients and ART patients. We will also investigate how factors like BMI, sex, and drug-drug interactions influence intracellular TFV-DP drug levels [32]. We will validate the RESTRICT assay to meet established Clinical Laboratory Improvement Amendments criteria to enable reporting of results to clients. The RESTRICT assay could be used to evaluate the role of adherence in treatment failure and emergence of drug resistance among people living with HIV. The RESTRICT assay could also be useful to screen eligible HIV vaccine trial candidates who have been taking PrEP in order to increase efficiency.

In conclusion, we evaluated a rapid, objective enzymatic assay for TFV-DP concentrations that correlate with long-term PrEP adherence. The RESTRICT assay identified participants with TFV-DP concentrations above the threshold for adequate adherence. The RESTRICT assay could be used to fill the gap of rapid long-term adherence measurement to promote more honest conversations about PrEP use and enable improved PrEP counselling. [7, 33, 34].

\section{Abbreviations \\ BMI: Body Mass Index; CFAR: Center for AIDS Research; DBS: Dried blood spot; HIV: Human immunodeficiency virus; IQR: Interquartile range; LC-MS/ MS: Liquid chromatography tandem mass spectrometry; PrEP: Pre-exposure prophylaxis; RBC: Red blood cell; RESTRICT: REverSe TRanscrlptase Chain Termination; RT: Reverse transcriptase; TDF: Tenofovir disoproxil fumarate; TFV: Tenofovir; TFV-DP: Tenofovir diphosphate.}

\section{Acknowledgements}

We are grateful for funding from the NIH (R01Al136648, R21Al127200, R01EB022630), the University of Washington CoMotion Innovation Gap Fund, and the Mistletoe Research Foundation. Part of this work was conducted using equipment in the Biochemical Diagnostics Foundry for Translational Research supported by the M.J Murdock Charitable Trust. Research reported in this publication was supported by the University of Washington/Fred Hutch Center for AIDS Research, an NIH-funded program under award number AI027757 which is supported by the following NIH Institutes and Centers: NIAID, NCl, $\mathrm{NIMH}, \mathrm{NIDA}, \mathrm{NICHD}, \mathrm{NHLBI}, \mathrm{NIA}$, NIGMS, NIDDK. We thank Lindsay Legg, MarkJason Cabudol, Kate Thanel, Abby Howell, Charlene Hayes, and Nina Kim at the CFAR for their help with participant recruitment and sample collection. We also thank Colleen Kimsey and Harald Haugen at the International Clinical Research Center at the University of Washington for their support with DBS sample transportation, storage, and shipping; and Yardpiroon Tawon at the PHPT-AMS laboratory at Chiang Mai University for DBS drug level measurements. We are also grateful for helpful conversations and support from Andrew Bender, Marta Fernandez-Suarez, Jay Rutherford, Rebecca Sandlin, Derin Sevenler, Mehmet Toner, and Jane Zhang. Figure 1 was created using BioRender.com.

\section{Authors' contributions}

AOO, JDP, PKD contributed to conceptualization and formulation of research goals and assay design. AOO and TJL performed RESTRICT experiments. AOO analyzed the data and prepared the figures. AOO, BPS, and JDP developed methodology and models for RESTRICT assay design. AOO, ARB, PKD and JDP contributed to study design, sample collection, and sample storage. TRC conducted data collection and analysis for LC-MS/MS analysis. AOO wrote the initial draft. AOO, JDP, and PKD revised the manuscript. All authors read and approved the final manuscript.

\section{Funding}

P.K Drain received grant funding from the NIH (R01Al136648, R21Al127200), J.D Posner received grant funding from the NIH (R01EB022630). P.K. Drain, A.O. Olanrewaju, and J.D Posner received grant funding from the University of Washington CoMotion Innovation Gap Fund. A.O. Olanrewaju received grant funding from the Mistletoe Research Foundation. For the remaining authors none were declared. Part of this work was conducted using equipment in the Biochemical Diagnostics Foundry for Translational Research supported by the M.J Murdock Charitable Trust. Research reported in this publication was supported by the University of Washington / Fred Hutch Center for AIDS Research, an $\mathrm{NIH}$-funded program under award number Al027757 which is supported by the following NIH Institutes and Centers: NIAID, NCI, NIMH, NIDA, NICHD, NHLBI, NIA, NIGMS, NIDDK.

Availability of data and materials

The data generated and analyzed during the current study are available in the Zenodo repository, https://doi.org/10.5281/zenodo.4244946.

\section{Declarations}

\section{Ethics approval and consent to participate}

All study participants were enrolled and sampled in accordance with the University of Washington/Fred Hutch Center for AIDS Research (CFAR) HIV Specimen Repository covered by the University of Washington's Institutional Review Board approval STUDY ID: STUDY00001258. All participants provided informed consent and samples were collected in association with study identifiers.

\section{Consent for publication}

Not applicable.

\section{Competing interests}

The authors declare that they have no competing interests.

\section{Author details}

${ }^{1}$ Department of Mechanical Engineering, University of Washington, Seattle, USA. ${ }^{2}$ Department of Global Health, Schools of Medicine and Public Health, University of Washington, Seattle, USA. ${ }^{3}$ Department of Materials Science and Engineering, University of Washington, Seattle, USA. ${ }^{4}$ PHPT/IRD 174, Department of Medical Technology, Faculty of Associated Medical Sciences, Chiang Mai University, Chiang Mai, Thailand. ${ }^{5}$ Harvard T.H. Chan School of Public Health, Harvard University, Boston, USA. ${ }^{6}$ Department of Molecular and Clinical Pharmacology, University of Liverpool, Liverpool, UK. ${ }^{7}$ Department of Chemical Engineering, University of Washington, Seattle, USA. 


\begin{abstract}
${ }^{8}$ Department of Family Medicine, University of Washington, Seattle, USA. ${ }^{9}$ Department of Medicine, School of Medicine, University of Washington, Seattle, USA. ${ }^{10}$ Department of Epidemiology, School of Public Health, University of Washington, Seattle, USA.
\end{abstract}

Received: 5 November 2020 Accepted: 3 April 2021 Published online: 15 April 2021

\section{References}

1. Cohen MS, Chen YQ, McCauley M, Gamble T, Hosseinipour MC, Kumarasamy $\mathrm{N}$, et al. Prevention of HIV-1 infection with early antiretroviral therapy. N Engl J Med. 2011;365(6):493-505.

2. Grant RM, Lama JR, Anderson PL, McMahan V, Liu AY, Vargas L, et al. Preexposure chemoprophylaxis for HIV prevention in men who have sex with men. N Engl J Med. 2010;363(27):2587-99.

3. Kashuba AD, Patterson KB, Dumond JB, Cohen MS. Pre-exposure prophylaxis for HIV prevention: how to predict success. The Lancet. 2012;379(9835):2409-11.

4. Fonner VA, Dalglish SL, Kennedy CE, Baggaley R, O'Reilly KR, Koechlin FM, et al. Effectiveness and safety of oral HIV preexposure prophylaxis for all populations. AIDS. 2016;30(12):1973-83.

5. van der Straten A, Van Damme L, Haberer JE, Bangsberg DR. Unraveling the divergent results of pre-exposure prophylaxis trials for HIV prevention. AIDS. 2012;26(7):F13.

6. Donnell D, Baeten JM, Bumpus NN, Brantley J, Bangsberg DR, Haberer JE, et al. HIV Protective efficacy and correlates of tenofovir blood concentrations in a clinical trial of PrEP for HIV prevention. J Acquir Immune Defic Syndr. 2014;66(3):340-8.

7. Celum CL, Mgodi N, Bekker L-G, Hosek S, Donnell D, Anderson PL, et al. PrEP adherence and effect of drug level feedback among young African women in HPTN 082. In: Proceedings of the 10th International AIDS Society Meeting, Mexico City, Mexico. 2019. p. 30-31.

8. Castillo-Mancilla JR, Haberer JE. Adherence measurements in HIV: new advancements in pharmacologic methods and real-time monitoring. Curr HIV/AIDS Rep. 2018;15(1):49-59.

9. Simoni JM, Kurth AE, Pearson CR, Pantalone DW, Merrill JO, Frick PA. Self-Report measures of antiretroviral therapy adherence: a review with recommendations for HIV research and clinical management. AIDS Behav. 2006;10(3):227-45.

10. Abaasa A, Hendrix C, Gandhi M, Anderson P, Kamali A, Kibengo F, et al. Utility of different adherence measures for PrEP: patterns and incremental value. AIDS Behav. 2018;22(4):1165-73.

11. McMahon JH, Jordan MR, Kelley K, Bertagnolio S, Hong SY, Wanke CA, et al. Pharmacy adherence measures to assess adherence to antiretroviral therapy: review of the literature and implications for treatment monitoring. Clin Infect Dis. 2011;52(4):493-506.

12. Anderson PL, Liu AY, Castillo-Mancilla JR, Gardner EM, Seifert SM, McHugh C, et al. Intracellular tenofovir-diphosphate and emtricitabinetriphosphate in dried blood spots following directly observed therapy. Antimicrob Agents Chemother. 2018;62(1):e01710-e1717.

13. Spinelli MA, Haberer JE, Chai PR, Castillo-Mancilla J, Anderson PL, Gandhi M. Approaches to objectively measure antiretroviral medication adherence and drive adherence interventions. Curr HIV/AIDS Rep. 2020;17(4):301-14.

14. Drain PK, Bardon AR, Simoni JM, Cressey TR, Anderson P, Sevenler D, et al. Point-of-care and near real-time testing for antiretroviral adherence monitoring to HIV treatment and prevention. Curr HIV/AIDS Rep. 2020;17(5):487-98.

15. Saag MS, Benson CA, Gandhi RT, Hoy JF, Landovitz RJ, Mugavero MJ, et al. Antiretroviral drugs for treatment and prevention of HIV infection in adults: 2018 recommendations of the international antiviral society-USA panel. JAMA. 2018;320(4):379-96.

16. Kearney BP, Flaherty JF, Shah J. Tenofovir Disoproxil Fumarate. Clin Pharmacokinet. 2004;43(9):595-612.

17. Hendrix CW, Andrade A, Bumpus NN, Kashuba AD, Marzinke MA, Moore $A$, et al. Dose frequency ranging pharmacokinetic study of tenofovir-emtricitabine after directly observed dosing in healthy volunteers to establish adherence benchmarks (HPTN 066). AIDS Res Hum Retroviruses. 2016;32(1):32-43.

18. Koenig HC, Mounzer K, Daughtridge GW, Sloan CE, Lalley-Chareczko L, Moorthy GS, et al. Urine assay for tenofovir to monitor adherence in real time to tenofovir disoproxil fumarate/emtricitabine as pre-exposure prophylaxis. HIV Med. 2017;18(6):412-8.

19. Drain PK, Kubiak RW, Siriprakaisil O, Klinbuayaem V, Quame-Amaglo J, Sukrakanchana P-O, et al. Urine tenofovir concentrations correlate with plasma and relate to tenofovir disoproxil fumarate adherence: a randomized, directly observed pharmacokinetic trial (TARGET Study). Clin Infect Dis. 2020;70(10):2143-51.

20. Castillo-Mancilla JR, Zheng J-H, Rower JE, Meditz A, Gardner EM, Predhomme J, et al. Tenofovir, emtricitabine, and tenofovir diphosphate in dried blood spots for determining recent and cumulative drug exposure. AIDS Res Hum Retroviruses. 2012;121010062750004.

21. Grant RM, Anderson PL, McMahan V, Liu A, Amico KR, Mehrotra M, et al. Uptake of pre-exposure prophylaxis, sexual practices, and HIV incidence in men and transgender women who have sex with men: a cohort study. Lancet Infect Dis. 2014;14(9):820-9.

22. Olanrewaju AO, Sullivan BP, Zhang JY, Bender AT, Sevenler D, Lo TJ, et al. Enzymatic assay for rapid measurement of antiretroviral drug levels. ACS Sens. 2020;5(4):952-9.

23. Cressey TR, Siriprakaisil O, Klinbuayaem V, Quame-Amaglo J, Kubiak RW, Sukrakanchana $\mathrm{P}$, et al. A randomized clinical pharmacokinetic trial of Tenofovir in blood, plasma and urine in adults with perfect, moderate and low PrEP adherence: the TARGET study. BMC Infect Dis. 2017;17:496.

24. Le Grice SFJ, Cameron CE, Benkovic SJ. [13] Purification and characterization of human immunodeficiency virus type 1 reverse transcriptase. Methods Enzymol. 1995;262:130-144.

25. Anderson PL, Glidden DV, Liu A, Buchbinder S, Lama JR, Guanira JV, et al. Emtricitabine-tenofovir concentrations and pre-exposure prophylaxis efficacy in men who have sex with men. Sci Transl Med. 2012;4(151):151.

26. Spinelli MA, Glidden DV, Rodrigues WC, Wang G, Vincent M, Okochi H, et al. Low tenofovir level in urine by a novel immunoassay is associated with seroconversion in a preexposure prophylaxis demonstration project. AIDS. 2019;33(5):867-72.

27. Pratt GW, Fan A, Melakeberhan B, Klapperich CM. A competitive lateral flow assay for the detection of tenofovir. Anal Chim Acta. 2018;1017:34-40.

28. Gandhi M, Bacchetti P, Rodrigues WC, Spinelli M, Koss CA, Drain PK, et al. Development and validation of an immunoassay for tenofovir in urine as a real-time metric of antiretroviral adherence. EClinicalMedicine. 2018;2-3:22-8.

29. Anderson PL. what can urine tell us about medication adherence? EClinicalMedicine. 2018;2:5-6.

30. Sidstedt M, Hedman J, Romsos EL, Waitara L, Wadsö L, Steffen CR, et al. Inhibition mechanisms of hemoglobin, immunoglobulin $\mathrm{G}$, and whole blood in digital and real-time PCR. Anal Bioanal Chem. 2018;410(10):2569-83.

31. Sidstedt $M$, Rådström $P$, Hedman J. PCR inhibition in $q P C R, d P C R$ and MPS-mechanisms and solutions. Anal Bioanal Chem. 2020;412:2009-23.

32. Coyle RP, Morrow M, Coleman SS, Gardner EM, Zheng J-H, Ellison L, et al. Factors associated with tenofovir diphosphate concentrations in dried blood spots in persons living with HIV. J Antimicrob Chemother. 2020;75(6):1591-8.

33. Bell KM, Haberer JE. Actionable adherence monitoring: technological methods to monitor and support adherence to antiretroviral therapy. Curr HIV/AIDS Rep. 2018;15(5):388-96.

34. Chaiyachati KH, Ogbuoji O, Price M, Suthar AB, Negussie EK, Bärnighausen T. Interventions to improve adherence to antiretroviral therapy: a rapid systematic review. AIDS. 2014;28:S187.

\section{Publisher's Note}

Springer Nature remains neutral with regard to jurisdictional claims in published maps and institutional affiliations. 